\title{
THE $Q$-MATRIX PROBLEM FOR MARKOV CHAINS
}

\author{
BY DAVID WILLIAMS
}

Communicated by D. Stroock, July 19, 1975

1. Let $I$ be a countable set. A ("standard") Markov transition function $(P(t))$ on $I$ may be regarded as a family $\left\{p_{i j}(\cdot): i, j \in I\right\}$ of functions on $[0, \infty)$ such that (for $i, j \in I$ and $s, t \in[0, \infty)$ )

$$
\begin{aligned}
& p_{i j}(t) \geqslant 0, \quad \sum_{k \in I} p_{i k}(t)=1, \\
& p_{i j}(s+t)=\sum_{k \in I} p_{i k}(s) p_{k j}(t), \lim _{u \downarrow 0} p_{i i}(u)=p_{i i}(0)=1
\end{aligned}
$$

If $(P(t))$ is a Markov transition function on $I$, the (Doob-Kolmogorov) limits

$$
-q_{i i}=\lim _{t \downarrow 0} t^{-1}\left[1-p_{i i}(t)\right], \quad q_{i j}=\lim _{t \downarrow 0} t^{-1} p_{i j}(t)
$$

exist in $[0, \infty]$ and satisfy

$$
\begin{aligned}
& 0 \leqslant q_{i j}<\infty \quad(i \neq j), \\
& \sum_{k \neq i} q_{i k} \leqslant-q_{i i} \leqslant \infty .
\end{aligned}
$$

The $I \times I$ matrix $Q=\left(q_{i j}\right)$ is called the $Q$-matrix of $(P(t))$ and we write $Q=$ $P^{\prime}(0)$.

The following theorem solves the $Q$-matrix problem for the case when all states are instantaneous $\left(q_{i i}=-\infty, \forall i\right)$.

THEOREM. Let $Q$ be an $I \times I$ matrix with

$$
q_{i i}=-\infty \quad(\forall i) ; \quad 0 \leqslant q_{i j}<\infty \quad(\forall i, j: i \neq j) .
$$

For $Q$ to be the $Q$-matrix of a Markov transition function $(P(t))$, it is necessary and sufficient that the following conditions (2) and (3) hold:

$$
\sum_{j \notin\{a, b\}} q_{a j} \wedge q_{b j}<\infty \quad(\forall a, b: a \neq b)
$$

(3) for every finite subset $H$ of $I$,

AMS (MOS) subject classifications (1970). Primary 60J 25, $60 \mathrm{~J} 40$.

Key words and phrases. Markov chain, $Q$-matrix, Ray-Knight compactification, Kendall's branching procedure. 


$$
\lim _{j} \inf \sum_{h \in H} q_{h j}=0
$$

[Note. The theorem remains true if "Markov" is replaced by "sub-Markov".] Acknowledgement. Paul Seymour (Swansea) sorted out the combinatorics of Lemma 2 and thus allowed me to give the above theorem in its "proper" form. My clumsy attempt at Lemma 2 worked only under a superfluous extra hypothesis.

2. Sketched proof of necessity of (2) and (3). Suppose that $Q$ is the $Q$ matrix of a Markov transition function $(P(t))$. Let $X$ be a Ray version of a Markov process (taking values in some Ray-Knight compactification $\bar{E}$ of $I$ ) with Ray transition function extending $(P(t))$. See Getoor [2] .

LEMMA 1 (LOCAL CHARACTER PROPERTY). Let $G$ be an open subset of $\bar{E}$ and let $h \in G \cap I$. Then (with $G^{c}$ denoting $\bar{E} \backslash G$ ),

$$
\sum_{j \in G^{c} \cap I} q_{h j}<\infty .
$$

Notation. Write $Q\left(h, G^{c} \cap I\right)$ for the sum (4).

Proof. Define

$$
\begin{aligned}
X_{t}^{G} & =X_{t}, \quad t<T_{G^{c}}=\inf \left\{s>0: X_{s} \in G^{c}\right\}, \\
& =\partial, \quad t \geqslant T_{G^{c}} .
\end{aligned}
$$

Then $X^{G}$ is a "standard" chain with "minimal" state-space $(G \cap I) \cup \partial$. By (DK1),

$$
\infty>\lim _{t \downarrow 0} t^{-1} P^{h}\left\{X_{t}^{G}=\partial\right\} \geqslant Q\left(h, G^{c} \cap I\right) .
$$

Condition (2) now follows from the Hausdorff property of the (metrizable) Ray-Knight topology. Condition (3) follows from the fact that under hypothesis (1) no point of $I$ can be isolated in $I$.

Notes. (i) The above arguments modernise those used to prove (2) and (3) in my 1967 paper [4].

(ii) Property (3) may be expressed in the following equivalent form: $\left(3^{*}\right)$ there exists an infinite subset $K$ of $I$ such that $Q(i, K \backslash i)<\infty, \forall i \in I$.

3. Proof of the sufficiency of conditions (2) and (3) is much harder. The main motivation comes from the probabilistic "branching" procedure which underlies Kendall's remarkable analytic construction of the "tree" process in the 1958 paper [3]. However, the "local character property" dominates the proof of "sufficiency" too.

We now assume that $Q$ is an $I \times I$ matrix satisfying (1), (2) and (3).

We say that $I$ is tree-labelled if $I$ is "labelled": $I=I_{0} \cup I_{1} \cup I_{2} \cup \cdots$, where $I_{0}=\{0\}$, and, for $n \in \mathrm{N}$,

$$
I_{n}=\left\{0 i_{1} i_{2} \cdots i_{n}: i_{1}, i_{2}, \ldots, i_{n} \in \mathrm{N}\right\} \text {. }
$$


Let $\pi$ be the immediate predecessor map of $I \backslash\{0\}$ to $I$ :

$$
\pi i=0 i_{1} i_{2} \cdots i_{n-1} \in I_{n-1} \text { when } i=0 i_{1} i_{2} \cdots i_{n} \in I_{n} \text {. }
$$

We can regard $\pi$ as being (rather than "inducing") a tree-labelling.

LEMma 2 (P. D. Seymour). There is a tree-labelling $\pi$ of I such that

$$
\underset{j \in \pi^{-1}\{i\}}{\lim \inf _{i j}} q_{i j}=0,
$$

where $Q^{-}$is the $I \times I$ matrix defined as follows:

$$
\begin{aligned}
q_{i j}^{\bar{j}} & =q_{i j} \quad \text { if } j \in\{i\} \cup \pi^{-1}\{i\}, \\
& =0 \quad \text { otherwise. }
\end{aligned}
$$

We now assume $I$ is already tree-labelled in accordance with Lemma 2.

4. For each $i$, we choose a certain special type of chain $X^{(i)}$ with minimal state-space $\{i\} \cup \pi^{-1}\{i\}$, with Markov transition function, and with $Q$-matrix $Q^{(i)}$ satisfying

$$
\begin{aligned}
& -q_{i i}^{(i)}=\infty, \quad q_{i j}^{(i)}=q_{i j} \quad\left(j \in \pi^{-1}\{i\}\right), \\
& -q_{j i}^{(i)}<\infty, \quad q_{j k}^{(i)}=0 \quad\left(j \in \pi^{-1}\{i\}, k \neq j\right) .
\end{aligned}
$$

It is important to realise that condition $4(\mathrm{i})$ is both necessary and sufficient for the existence of a chain $X^{(i)}$ with Markov transition function and with $Q$-matrix satisfying conditions (5). It should also be realised that $X^{(i)}$ is necessarily a complicated chain with infinitely many fictitious states.

5. Kendall's branching procedure. The chain $X^{(0)}$ has minimal state-space $\{0,01,02, \ldots\}$. The state 01 is stable for $X^{(0)}$ with rate $a_{01}$ (say). During each (exponentially distributed) visit by $X^{(0)}$ to 01 , replace $X^{(0)}$ by a chain on $\{01\} \cup \pi^{-1}\{01\}$ with the $P^{(01)}$ law of $X^{(01)}$. (In effect, this last-mentioned chain is killed at rate $a_{01}$.) After modifying $X^{(0)}$ on its "01-intervals", we obtain a chain on $\{0,01\} \cup\{02,03, \ldots\} \cup\{011,012, \ldots\}$ for which the first two states (and only these) are instantaneous.

By proceeding in the obvious inductive fashion, we can-provided that we have chosen the $X^{(i)}$ with sufficient care-obtain a "projective limit" chain $X^{-}$ with $Q$-matrix $Q^{-}$. Since $Q$ differs only "finitely" from $Q^{-}$in the sense of 4(ii), it is easy (with the same proviso as above) to extend the Levy system of $X^{-}$so as to produce a chain $X$ with the desired $Q$-matrix $Q$.

The way to choose the $X^{(i)}$ will be explained in the "full" version of this work. The existence of the limit chain $X^{-}$is proved by the method in Freedman's book [1].

\section{REFERENCES}

1. D. Freedman, Approximating Markov chains, Holden-Day, San Francisco, Calif., 1972. 
2. R. K. Getoor, Markov processes: Ray processes and right processes, Lecture Notes in Math., vol. 440, Springer-Verlag, Berlin and New York, 1975.

3. D. G. Kendall, A totally unstable denumerable Markov process, Quart. J. Math. Oxford Ser. (2) 9 (1958), 149-160. MR 20 \#4319.

4. D. Williams, $A$ note on the $Q$-matrices of Markov chains, Z. Wahrscheinlichkeitstheorie und Verw. Gebiete 7 (1967), 11.6-121. MR 37 \#2326.

DEPARTMENT OF MATHEMATICS, SINGLETON PARK, SWANSEA SA2 8PP, GREAT BRITAIN 